\title{
Autopercepção de pessoas acometidas pela hanseníase sobre sua saúde bucal e necessidade de tratamento
}

\author{
Self-perception of people afflicted with leprosy \\ regarding their oral health and the need for treatment
}

Janaína Rocha de Sousa Almeida ${ }^{1}$

Carlos Henrique Alencar ${ }^{2}$

Jaqueline Caracas Barbosa ${ }^{3}$

Aldo Angelim Dias ${ }^{4}$

Maria Eneide Leitão de Almeida ${ }^{5}$

${ }^{1}$ Centro de Saúde da Família Anastácio Magalhães, Prefeitura Municipal de Fortaleza. Rua Delmiro de Farias, Rodolfo Teófilo. 60000-000 Fortaleza CE.

drajanainarocha@hotmail.com ${ }^{2}$ Departamento de Saúde Comunitária, Faculdade de Medicina, Universidade Federal do Ceará.

${ }^{3}$ Departamento de Saúde Comunitária, Faculdade de Medicina, Universidade Federal do Ceará.

${ }^{4}$ Curso de Odontologia, Universidade de Fortaleza. ${ }^{5}$ Departamento de Clínica Odontológica, Centro de Ciências da Saúde,

Universidade Federal do Ceará.

\begin{abstract}
Leprosy is an infectious disease that has an impact from a physical, social and psychological standpoint. The scope of this study was to assess the self-perception on oral health and need for treatment in leprosy patients in the city of Fortaleza, State of Ceará, Brazil. This is a crosssectional and descriptive study, where 100 leprosy patients were given a semi-structured questionnaire to fill out. The results of the bivariate analysis between need for treatment and socioeconomic characteristics showed that only education revealed a statistical association ( $p=0.000$ ). An association was verified between the classification of oral health and self-perception of need for treatment ( $p=0.05$ ). With respect to the selfperception of oral health, 36\% of the researched subjects classified their oral health as good. The self-perception of leprosy patients regarding their oral health and need for treatment, together with a clinical evaluation, should serve as a guide for the drafting of public policies that aim to foment more effective dental treatment for these patients. Key words Leprosy, Self-image, Oral health
\end{abstract}

Resumo A hanseníase é uma doença infecciosa que produz impacto do ponto de vista físico, social e psicológico. O propósito deste estudo foi avaliar a autopercepção sobre saúde bucal e a necessidade de tratamento em pacientes com hanseniase no Municipio de Fortaleza (CE), Brasil. Trata-se de um estudo transversal e descritivo, onde 100 pacientes com hanseníase foram submetidos a questionário semiestruturado. Os resultados da análise bivariada entre necessidade de tratamento e características socioeconômicas mostrou que apenas a escolaridade apresentou associação estatística ( $p=0,000)$. Verificou-se associação entre a classificação da saúde bucal e autopercepção da necessidade de tratamento $(p=0,05)$. Com relação à autopercepção em saúde bucal, 36\% dos sujeitos pesquisados classificaram sua saúde bucal como boa. A autopercepção do paciente com hanseníase quanto a sua saúde bucal e necessidade de tratamento deve, juntamente com uma avaliação clínica, servir de guia para a execução de políticas públicas que visem a favorecer um tratamento odontológico mais efetivo para esses pacientes. Palavras-chave Hanseníase, Autoimagem, Saúde Bucal 


\section{Introdução}

A hanseníase ou doença de Hansen é uma doença bastante estigmatizada, contagiosa, mutilante e que provoca rejeição e preconceito ao paciente portador, este fato é gerado principalmente pela falta de informação da população, o que pode originar na sua exclusão da sociedade ${ }^{1-5}$, sendo que sua evolução pode ocasionar graves consequências, como lesões incapacitantes, aumentado assim as repercussões sociais ${ }^{6,7}$.

Trata-se de uma das principais doenças infecciosas que produz impacto não apenas do ponto de vista físico como também social e psicológico $^{6,8-11}$. Apesar de todo o trabalho realizado com o objetivo de controlar a hanseníase, tentando desmistificar alguns conceitos errôneos, incentivar o correto diagnóstico e execução do tratamento de poliquimioterapia, o medo com relação à doença ainda é evidente ${ }^{6}$.

A hanseníase é uma doença infecciosa crônica causada pela bactéria intracelular e acidorresistente Mycobacterium leprae ${ }^{4,12}$ que acomete preferencialmente pele e nervos periféricos, com um grande potencial para desenvolver incapacidades físicas, que podem evoluir para deformidades visíveis, mais comuns em mãos, pés e áreas da face ${ }^{13,14}$. Sua transmissão acontece pelas vias aéreas superiores ${ }^{8,15}$. A hanseníase apresenta lesões de pele com diminuição ou ausência de sensibilidade, sendo as mais comuns as manchas as pigmentares, as placas, as infiltrações, os tubérculos e os nódulos. As lesões podem acometer qualquer local do corpo, inclusive a mucosa nasal e, mais raramente, a cavidade oral ${ }^{16}$. A alteração de sensibilidade diferencia as lesões hansênicas das outras dermatológicas ${ }^{8}$. A classificação de Madri (1953) divide a hanseníase de acordo com sua forma clínica em indeterminada, estágio inicial da doença; tuberculoide, contenção da multiplicação bacilar; dimorfos, forma de instabilidade imunológica; e virchowianos, quando ocorre maior multiplicação e disseminação da doença.

Avaliando a relação da saúde bucal com a hanseníase, verifica-se que as infecções odontológicas podem ser fatores desencadeantes de episódios de reações hansênicas, que são períodos de inflamação aguda no curso de uma doença crônica que podem afetar os nervos ${ }^{17}$. Esta inflamação aguda é causada pela atuação do sistema imunológico do hospedeiro que ataca o $\mathrm{Myco}$ bacterium leprae ${ }^{17}$. Doenças gengivais e periodontais estão entre os fatores bucais mais prováveis na ocorrência dos episódios reacionais ${ }^{18}$.
Do ponto de vista clínico, as principais manifestações bucais associadas à hanseníase referemse a alterações gengivais na porção anterior da maxila, palato duro e mole, úvula e língua ${ }^{19,20}$, entretanto, não existem lesões patognomônicas na cavidade oral ${ }^{16,20-22}$. Quanto ao grau de envolvimento do palato, as lesões estão muito relacionadas com a duração da doença, representando importante marcador clínico aninhado a outras manifestações sistêmicas ${ }^{16}$. Ressalta-se que atualmente o tratamento precoce com a poliquimioterapia reduz a incidência de lesões orais, tornando-as mais raras ${ }^{16,23,24}$. A manutenção de infecções orais, no entanto, pode levar a reações, dificultando o tratamento do paciente ${ }^{23}$. Em razão da possibilidade de ocorrência destas lesões, a avaliação sistemática do padrão das condições bucais é recomendada na rotina dos serviços ${ }^{21}$.

Poucos são os estudos a respeito da saúde bucal dos pacientes com hanseníase ${ }^{16,22,25}$, e mais rara ainda é a avaliação da autopercepção destes com a própria. Nesse sentido, este estudo se propõe conhecer as percepções desses pacientes com relação à sua saúde bucal e à necessidade de tratamento, podendo servir como referência para a elaboração de políticas de saúde para esses indivíduos.

A autopercepção é a interpretação que uma pessoa faz do seu estado de saúde, contribuindo para isso os mais diversos fatores, sejam eles direta ou indiretamente relacionados, sendo considerada uma variável multidimensional. Uma complexidade de fatores influencia nesse julgamento, dentre eles as características demográficas, como idade, sexo, raça e fatores de predisposição, como escolaridade e acesso a informações sobre cuidados preventivos, que podem influenciar na predisposição para o uso de serviços odontológicos e, consequentemente, na autopercepção da saúde bucal ${ }^{26-30}$.

A autopercepção está associada a fatores objetivos e subjetivos. Dentre os parâmetros de avaliação objetiva, realizada pelo cirurgião-dentista, podem-se destacar os índices como CPO que avalia o número de dentes cariados, perdidos e obturados; índices que avaliam a condição do periodonto, como o índice periodontal comunitário (CPI) e índice de perda de inserção $(\mathrm{PIP})^{26,29,30}$. Como parâmetros subjetivos, temse a autoavaliação da necessidade de tratamento odontológico, sensibilidade dolorosa nos dentes e gengivas, aparência bucal, da mastigação, da fala e dos relacionamentos sociais em função das condições bucais ${ }^{26,29-34}$.

A autopercepção de saúde bucal não está dissociada da saúde em geral, recebendo a influên- 
cia da presença de doenças sistêmicas e da saúde mental ${ }^{26}$. Desse modo, o propósito do presente estudo foi avaliar a autopercepção sobre a saúde bucal e a necessidade de tratamento de pacientes com hanseníase no Município de Fortaleza (CE), Brasil.

\section{Métodos}

Este estudo caracteriza-se como transversal e descritivo, tendo o período de coleta iniciado em dezembro de 2009 e finalizando em julho de 2010. Foram entrevistados 100 pacientes com hanseníase, acompanhados no ambulatório de dermatologia do Hospital Universitário Walter Cantídio, da Universidade Federal do Ceará, Município de Fortaleza (CE), Brasil. Este hospital é uma das unidades de referência para o atendimento de pacientes com hanseníase no Estado do Ceará. Como critérios de inclusão no estudo, o paciente deveria residir no Município de Fortaleza e ter idade maior ou igual a 15 anos. Foram entrevistados todos os pacientes com hanseníase que estavam em acompanhamento, seja no período de tratamento ou pós-tratamento, e dentro dos critérios de inclusão.

Utilizou-se para a coleta dos dados um questionário adaptado do Projeto Condições de Saúde Bucal da População Brasileira-SB Brasil 2002/ 2003, que avalia a situação socioeconômica, o acesso a serviços odontológicos e a autopercepção em saúde bucal, além das condições de saúde bucal (cárie, doença periodontal, necessidade de tratamento, uso e necessidade de prótese e alterações de tecidos moles) ${ }^{35}$. Foram incluídas nesse questionário perguntas específicas relacionadas à hanseníase, avaliando-se as interações desta doença com a autopercepção da saúde bucal. Foi realizado um estudo-piloto em dez portadores de hanseníase que não fizeram parte da referida pesquisa, com o objetivo de realizar os devidos ajustes ao instrumento de pesquisa. Todas as entrevistas foram realizadas por uma só pesquisadora no próprio hospital onde esses pacientes eram acompanhados.

Inicialmente foi realizada a análise descritiva da população estudada e, em seguida, foram avaliadas as relações estatísticas entre as diversas variáveis. A primeira variável dependente avaliada foi a autopercepção da necessidade de tratamento odontológico, por meio do questionamento: "Considera que necessita de tratamento atualmente?”. Como variáveis independentes, foram empregadas as características demográfi- cas, situação socioeconômica, aspectos relacionados à hanseníase e condições da saúde bucal. A segunda variável dependente avaliada foram os prejuízos causados pela doença à sua saúde bucal, mediante o questionamento: "A doença hanseníase trouxe algum prejuízo para sua saúde bucal? Se sim qual?". As variáveis independentes analisadas foram dificuldade de higienização, classificação da saúde bucal, classificação da aparência dos dentes e gengivas, classificação da mastigação, classificação da fala, relação da saúde bucal e relacionamento com outras pessoas e quantidade de dor nos dentes e gengivas nos últimos seis meses.

A análise estatística foi realizada de forma descritiva, ressaltando-se as dimensões mais relevantes. Os dados foram analisados pelo programa Stata 11, tendo-se aplicado o teste de Pearson e de Fisher para verificar associação entre as variáveis, considerando-se significativo com o valor $\mathrm{p}<0,05$.

Este estudo teve início após a aprovação pelo Comitê de Ética em Pesquisa da Universidade Federal do Ceará e foi conduzido conforme os princípios éticos da Declaração de Helsinque, contidos na Resolução n. 196/96 ${ }^{36}$ do Conselho Nacional de Saúde, que regula investigações realizadas em seres humanos.

\section{Resultados}

Nos 100 pacientes com hanseníase examinados, a idade variou de 25 a 84 anos, apresentando média de 49,32 anos e desvio-padrão de 13,82 e foi observado que a maioria (52\%) era do sexo masculino. Quanto às características socioeconômicas, prevaleceu uma baixa escolaridade, pois $48 \%$ apresentava o ensino fundamental incompleto. Referente à moradia, 75\% tinham casa própria, contudo $29 \%$ dos entrevistados não possuía renda pessoal.

$\mathrm{Na}$ caracterização da doença hanseníase, investigou-se há quanto tempo o paciente descobriu que tinha a doença, tendo-se verificado que para a maioria (28\%) foi entre um e dois anos. A forma virchowiana foi encontrada em $57 \%$ dos portadores. Episódios de reações hansênicas foram relatados por $58 \%$ dos entrevistados.

A necessidade atual de tratamento dentário foi referida por $73 \%$ dos entrevistados, $25 \%$ acharam que não precisavam e $2 \%$ não souberam responder.

Os resultados da análise bivariada entre autopercepção da necessidade de tratamento e ca- 
racterísticas socioeconômicas e caracterização da hanseníase mostraram que apenas a escolaridade apresentou associação estatística $(\mathrm{p}=0,000)$, como pode ser observado na Tabela 1 , onde se verifica que, quanto maior o grau de escolaridade, maior a percepção da necessidade de tratamento. Não foi verificada associação estatística entre a autopercepção da necessidade de tratamento e os demais fatores, como: gênero ( $\mathrm{p}=$ $0,895)$, tipo de moradia ( $\mathrm{p}=0,978)$, renda pessoal $(p=0,236)$, tempo da doença $(p=0,771)$ e forma de hanseníase $(\mathrm{p}=0,928)$.

No que concerne à autopercepção da saúde bucal, 36\% dos sujeitos pesquisados a classificaram como boa. Quanto à aparência de dentes e gengivas, foi considerada boa para $44 \%$ dos pacientes.
Os indivíduos estudados consideraram sua mastigação e a fala como boas em $49 \%$ e $53 \%$, respectivamente. No que se refere ao relacionamento com outras pessoas, $36 \%$ dos portadores de hanseníase consideraram que a saúde bucal afeta muito o relacionamento interpessoal. Mais da metade $(58 \%)$ dos entrevistados não relatou dor nos dentes ou gengivas nos últimos seis meses.

A análise bivariada mostrou associação estatística entre a classificação da saúde bucal e a autopercepção da necessidade de tratamento $(\mathrm{p}=$ 0,050), onde quanto pior foi a autopercepção sobre a saúde bucal maior era a percepção de necessidade de tratamento odontológico, conforme Tabela 2. Este fato também foi verificado, quando se observou a associação entre a autopercepção de necessidade de tratamento e a clas-

Tabela 1. Caracterização socioeconômica dos pacientes com hanseníase, caracterização da hanseníase e sua correlação com a necessidade de tratamento. Fortaleza (CE), 2010.

\begin{tabular}{|c|c|c|c|c|c|c|c|c|}
\hline \multirow{3}{*}{ Variáveis } & \multirow{3}{*}{ Total } & \multicolumn{6}{|c|}{ Com Necessidade de Tratamento } & \multirow{3}{*}{ Valor $\mathbf{p}$} \\
\hline & & \multicolumn{2}{|r|}{ Sim } & \multicolumn{2}{|r|}{ Não } & \multicolumn{2}{|c|}{ Não sabe } & \\
\hline & & $\mathbf{n}$ & $\%$ & $\mathrm{n}$ & $\%$ & n & $\%$ & \\
\hline Gênero & & & & & & & & 0,895 \\
\hline Masculino & 52 & 39 & $75,00 \%$ & 12 & $23,08 \%$ & 1 & $1,92 \%$ & \\
\hline Feminino & 48 & 34 & $70,83 \%$ & 13 & $27,08 \%$ & 1 & $2,08 \%$ & \\
\hline Escolaridade & & & & & & & & 0,000 \\
\hline Sem Escolaridade & 10 & 3 & $30,00 \%$ & 5 & $50,00 \%$ & 2 & $20,00 \%$ & \\
\hline Pré-escolar & 7 & 3 & $42,86 \%$ & 4 & $57,14 \%$ & 0 & $0,00 \%$ & \\
\hline Fundamental Incompleto & 48 & 38 & $79,17 \%$ & 10 & $20,83 \%$ & 0 & $0,00 \%$ & \\
\hline FundamentalCompleto & 13 & 12 & $92,31 \%$ & 1 & $7,69 \%$ & 0 & $0,00 \%$ & \\
\hline Ensino Médio & 20 & 17 & $85,00 \%$ & 3 & $15,00 \%$ & 0 & $0,00 \%$ & \\
\hline Ensino Superior & 2 & 0 & $0,00 \%$ & 2 & $100 \%$ & 0 & $0,00 \%$ & \\
\hline Moradia & & & & & & & & 0,978 \\
\hline Própria & 75 & 55 & $73,33 \%$ & 18 & $24,00 \%$ & 2 & $2,67 \%$ & \\
\hline Alugada/Outros & 25 & 18 & $72,00 \%$ & 7 & $28,00 \%$ & 0 & $0,00 \%$ & \\
\hline Renda pessoal & & & & & & & & 0,236 \\
\hline Sem Renda & 29 & 25 & $86,21 \%$ & 4 & $13,79 \%$ & 0 & $0,00 \%$ & \\
\hline < de 1 sal. Mínimo & 12 & 11 & $91,67 \%$ & 1 & $8,33 \%$ & 0 & $0,00 \%$ & \\
\hline 1 a 2 sal. Mínimos & 48 & 28 & $58,33 \%$ & 18 & $37,50 \%$ & 2 & $4,17 \%$ & \\
\hline 3 a 5 sal. Mínimos & 9 & 8 & $88,89 \%$ & 1 & $11,11 \%$ & 0 & $0,00 \%$ & \\
\hline 6 ou mais sal. Mín. & 2 & 1 & $50,00 \%$ & 1 & $50,00 \%$ & 0 & $0,00 \%$ & \\
\hline Tempo da Doença & & & & & & & & 0,771 \\
\hline$<$ de 6 meses & 17 & 11 & $64,71 \%$ & 5 & $29,41 \%$ & 1 & $5,88 \%$ & \\
\hline 6 meses a 1 ano & 17 & 14 & $82,35 \%$ & 3 & $17,65 \%$ & 0 & $0,00 \%$ & \\
\hline 1 a 2 anos & 28 & 20 & $71,43 \%$ & 8 & $28,57 \%$ & 0 & $0,00 \%$ & \\
\hline 3 a 5 anos & 22 & 15 & $68,18 \%$ & 6 & $27,27 \%$ & 1 & $4,55 \%$ & \\
\hline 6 a 10 anos & 11 & 10 & $90,91 \%$ & 1 & $9,09 \%$ & 0 & $0,00 \%$ & \\
\hline > De 10 anos & 5 & 3 & $60,00 \%$ & 2 & $40,00 \%$ & 0 & $0,00 \%$ & \\
\hline Tipo de Hanseníase & & & & & & & & 0,928 \\
\hline Indeterminada & 4 & 3 & $75,00 \%$ & 1 & $25,00 \%$ & 0 & $0,00 \%$ & \\
\hline Dimorfa & 33 & 25 & $75,76 \%$ & 8 & $24,24 \%$ & 0 & $0,00 \%$ & \\
\hline Tuberculoide & 6 & 5 & $83,33 \%$ & 1 & $16,67 \%$ & 0 & $0,00 \%$ & \\
\hline Virchowiana & 57 & 40 & $70,18 \%$ & 15 & $26,32 \%$ & 2 & $3,50 \%$ & \\
\hline
\end{tabular}


sificação da aparência dos dentes e gengivas $(\mathrm{p}=$ $0,000)$, uma vez que pacientes que classificaram sua aparência como péssima, ruim e regular percebem maior necessidade de realizar tratamento odontológico. Não foi verificada associação entre a autopercepção da necessidade de tratamento odontológico e a autopercepção sobre mastigação $(p=0,256)$, fala $(p=0,702)$, relacionamento interpessoal $(p=0,541)$ e dor nos dentes e gengivas $(\mathrm{p}=0,147)$, como pode ser observado na Tabela 2.

No que se refere ao acesso aos serviços odontológicos, $98 \%$ já foram ao dentista alguma vez na vida, entretanto, somente $30 \%$ receberam informações sobre como evitar problemas bucais.

Quando questionados se a doença hanseníase trouxe alguma dificuldade para realizar a higiene oral, $87 \%$ relataram não sentir problemas.

Tabela 2. Caracterização dos pacientes com hanseníase, de acordo com a autopercepção de sua saúde bucal e a correlação com a autopercepção da necessidade de realizar tratamento odontológico. Fortaleza (CE) - 2010.

\begin{tabular}{|c|c|c|c|c|c|c|c|c|}
\hline \multirow{3}{*}{ Variáveis } & \multirow{3}{*}{ Total } & \multicolumn{6}{|c|}{ Com Necessidade de Tratamento } & \multirow{3}{*}{ Valor $\mathrm{p}$} \\
\hline & & \multicolumn{2}{|r|}{ Sim } & \multicolumn{2}{|r|}{ Não } & \multicolumn{2}{|c|}{ Não sabe } & \\
\hline & & $\mathbf{n}$ & $\%$ & n & $\%$ & $\mathbf{n}$ & $\%$ & \\
\hline Autopercepção de saúde bucal & & & & & & & & 0,054 \\
\hline Não sabe & 1 & 1 & $100 \%$ & 0 & $0,00 \%$ & 0 & $0,00 \%$ & \\
\hline Péssima & 7 & 7 & $100 \%$ & 0 & $0,00 \%$ & 0 & $0,00 \%$ & \\
\hline Ruim & 14 & 13 & $92,86 \%$ & 1 & $7,14 \%$ & 0 & $0,00 \%$ & \\
\hline Regular & 34 & 26 & $76,47 \%$ & 6 & $17,65 \%$ & 2 & $5,88 \%$ & \\
\hline Boa & 36 & 23 & $63,89 \%$ & 13 & $36,11 \%$ & 0 & $0,00 \%$ & \\
\hline Ótima & 8 & 3 & $37,50 \%$ & 5 & $62,50 \%$ & 0 & $0,00 \%$ & \\
\hline Autopercepção da aparência de dentes e gengivas & & & & & & & & 0,000 \\
\hline Não sabe & 2 & 1 & $50,00 \%$ & 1 & $50,00 \%$ & 0 & $0,00 \%$ & \\
\hline Péssima & 9 & 9 & $100 \%$ & 0 & $0,00 \%$ & 0 & $0,00 \%$ & \\
\hline Ruim & 14 & 12 & $85,71 \%$ & 2 & $14,29 \%$ & 0 & $0,00 \%$ & \\
\hline Regular & 27 & 25 & $92,59 \%$ & 2 & $7,41 \%$ & 0 & $0,00 \%$ & \\
\hline Boa & 44 & 26 & $59,09 \%$ & 17 & $38,64 \%$ & 1 & $2,27 \%$ & \\
\hline Ótima & 4 & 0 & $0,00 \%$ & 3 & $75,00 \%$ & 1 & $25,00 \%$ & \\
\hline Autopercepção da mastigação & & & & & & & & 0,256 \\
\hline Não sabe & 1 & 1 & $100 \%$ & 0 & $0,00 \%$ & 0 & $0,00 \%$ & \\
\hline Péssima & 5 & 5 & $100 \%$ & 0 & $0,00 \%$ & 0 & $0,00 \%$ & \\
\hline Ruim & 22 & 21 & $95,45 \%$ & 1 & $4,55 \%$ & 0 & $0,00 \%$ & \\
\hline Regular & 10 & 7 & $70,00 \%$ & 3 & $30,00 \%$ & 0 & $0,00 \%$ & \\
\hline Boa & 49 & 31 & $63,27 \%$ & 16 & $32,46 \%$ & 2 & $4,08 \%$ & \\
\hline Ótima & 13 & 8 & $61,54 \%$ & 5 & $38,46 \%$ & 0 & $0,00 \%$ & \\
\hline Autopercepção da fala & & & & & & & & 0,702 \\
\hline Não sabe & 0 & 0 & $0,00 \%$ & 0 & $0,00 \%$ & 0 & $0,00 \%$ & \\
\hline Péssima & 1 & 1 & $100 \%$ & 0 & $0,00 \%$ & 0 & $0,00 \%$ & \\
\hline Ruim & 16 & 13 & $81,25 \%$ & 2 & $12,50 \%$ & 1 & $6,25 \%$ & \\
\hline Regular & 14 & 11 & $78,57 \%$ & 3 & $21,43 \%$ & 0 & $0,00 \%$ & \\
\hline Boa & 53 & 35 & $66,03 \%$ & 17 & $32,08 \%$ & 1 & $1,89 \%$ & \\
\hline Ótima & 16 & 13 & $81,25 \%$ & 3 & $18,75 \%$ & 0 & $0,00 \%$ & \\
\hline Relacionamento interpessoal & & & & & & & & 0,541 \\
\hline Não sabe & 5 & 2 & $40,00 \%$ & 3 & $60,00 \%$ & 0 & $0,00 \%$ & \\
\hline Não afeta & 28 & 20 & $71,43 \%$ & 7 & $25,00 \%$ & 1 & $3,57 \%$ & \\
\hline Afeta pouco & 11 & 9 & $81,82 \%$ & 2 & $18,18 \%$ & 0 & $0,00 \%$ & \\
\hline Afeta mais ou menos & 20 & 13 & $65,00 \%$ & 7 & $35,00 \%$ & 0 & $0,00 \%$ & \\
\hline Afeta muito & 36 & 29 & $80,55 \%$ & 6 & $16,67 \%$ & 1 & 2,78 & \\
\hline Autopercepção da dor nos dentes e gengivas & & & & & & & & 0,147 \\
\hline Nenhuma dor & 58 & 39 & $67,25 \%$ & 18 & $31,03 \%$ & 1 & $1,72 \%$ & \\
\hline Pouca dor & 22 & 18 & $81,82 \%$ & 4 & $18,18 \%$ & 0 & $0,00 \%$ & \\
\hline Média dor & 7 & 4 & $57,14 \%$ & 3 & $42,86 \%$ & 0 & $0,00 \%$ & \\
\hline Muita dor & 13 & 12 & $92,31 \%$ & 0 & $0,00 \%$ & 1 & $7,69 \%$ & \\
\hline
\end{tabular}


Este estudo não verificou associação entre a necessidade de realizar tratamento odontológico e o fato de receber orientações sobre como evitar os problemas bucais $(\mathrm{p}=0,455)$ ou ter dificuldade de realizar a higiene oral $(\mathrm{p}=0,106)$.

Foi questionado se a hanseníase trouxe algum prejuízo para a saúde bucal do paciente e $76 \%$ relataram que não verificaram prejuízo algum. Dentre os $18 \%$ que relataram ter tido prejuízo, foram citados: os dentes ficaram fracos $(27,80 \%)$, inchaço na boca $(16,70 \%)$, inflamação nos lábios e gengiva $(16,70 \%)$, dentes sensíveis $(16,70 \%)$, dores nos dentes (5,55\%), boca ressecada (5,55\%), ardência na boca $(5,55 \%)$ e dificuldade de segurar a escova dentária $(5,55 \%)$. Dentre os entrevistados $6 \%$ respondeu não saber se existiu algum prejuízo para sua saúde bucal.

Os pacientes com hanseníase que não apresentaram dificuldade de higienização bucal relatam em sua maioria $(81,61 \%)$ que a doença não trouxe prejuízo para sua saúde bucal $(p=0,003)$, conforme mostra a Tabela 3. Não foi verificada associação estatística entre o prejuízo à saúde bucal causado pela hanseníase e a classificação da saúde bucal $(\mathrm{p}=0,334)$, aparência dos dentes e gengivas $(p=0,764)$, mastigação $(p=0,520)$ e fala $(p=0,941)$, assim como não houve associação com a quantidade de dor nos dentes ou gengivas sentida nos últimos seis meses $(\mathrm{p}=0,529)$. Ressalta-se que quanto mais tardio o diagnóstico e o início do tratamento, maiores as chances de surgir alguma sequela neuromotora que possa proporcionar dificuldades na realização dos hábitos de higiene oral, o que poderia acarretar problemas com a saúde bucal desses pacientes.

\section{Discussão}

Atualmente, para a verificação da saúde bucal e da necessidade de tratamento dos pacientes, utilizam-se, além dos dados quantitativos obtidos pela visão do profissional, indicadores qualitativos obtidos de acordo com a autopercepção do paciente ${ }^{31}$, conseguindo-se, desta forma, ter uma percepção mais complexa dos problemas de cada individuo com suas devidas particularidades.

Quanto à caracterização dos portadores de hanseníase, na maioria das regiões do mundo, a incidência da doença é maior nos homens do que nas mulheres ${ }^{9,37}$, considerando, geralmente, o risco de exposição como fator responsável ${ }^{38}$. Nesta pesquisa também foi observada maior prevalência de homens vivendo com a referida doença.

O predomínio de portadores de hanseníase com poucos anos de escolaridade foi verificado em $41,8 \%$ dos casos na pesquisa de Santana et al. ${ }^{39}$, fato também verificado nesta pesquisa, no qual $48 \%$ dos entrevistados possuía no máximo o ensino fundamental incompleto. Amaral e Lana $^{40}$ relatam que a baixa escolaridade é um fator de risco para o desenvolvimento de formas incapacitantes da doença. O grau de escolaridade é também um fator preditor da autopercepção da saúde bucal e da necessidade de tratamento destes pacientes, pois, quanto maior a escolaridade melhor a percepção de sua saúde bucal e maior a percepção para a realização de tratamento odontológico ${ }^{26,30}$.

Observou-se associação entre a autopercepção da necessidade de tratamento e a escolaridade do paciente, em que um maior grau desta favorece a percepção da pessoa quanto à sua necessidade de tratamento.

Em estudo de Kerr-Pontes et al. ${ }^{41}$, foi verificado que no Ceará a hanseníase está, supostamente, associada ao elevado nível de pobreza e urbanização descontrolada, causando uma polarização da doença, sendo mais susceptível a população que vive com grande dificuldade econômica. As cidades que apresentaram maior incidência foram aquelas com maior urbanização e desenvolvimento econômico, cidades estas que, por sua vez, apresentam pior distribuição de renda entre

Tabela 3. Associação entre dificuldade de higienização bucal e os prejuízos trazidos para a saúde bucal em decorrência da hanseníase. Fortaleza (CE), 2010.

\begin{tabular}{|c|c|c|c|c|c|c|c|}
\hline \multirow{3}{*}{ Dificuldade de higienização bucal } & \multicolumn{6}{|c|}{ Prejuízos causados pela hanseníase } & \multirow{3}{*}{ Total } \\
\hline & \multicolumn{2}{|r|}{ Sim } & \multicolumn{2}{|c|}{ Não } & \multicolumn{2}{|c|}{ Não sabe } & \\
\hline & $\mathbf{n}$ & $\%$ & $\mathbf{n}$ & $\%$ & n & $\%$ & \\
\hline Sim & 6 & $46,15 \%$ & 5 & $38,46 \%$ & 2 & $15,38 \%$ & 13 \\
\hline Não & 12 & $13,79 \%$ & 71 & $81,61 \%$ & 4 & $4,60 \%$ & 87 \\
\hline Total & 18 & $18,00 \%$ & 76 & $76,00 \%$ & 6 & $6,00 \%$ & 100 \\
\hline
\end{tabular}

Valor de $\mathrm{p}=0,003$ 
a população, sendo, no Ceará, a distribuição da hanseníase bastante heterogênea. Corrobora com este achado o fato de $29 \%$ dos portadores de hanseníase não possuírem renda pessoal.

Quanto à classificação da forma clínica da hanseníase, Santana et al. ${ }^{38}$ observaram que 58,2\% apresentavam a forma tuberculoide, $27,3 \%$ a dimorfa, $12,7 \%$ a virchowiana e $1,8 \%$ a indeterminada. Já a população desta pesquisa apresentou $57 \%$ com a forma virchowiana, corroborando com os dados de Sobrinho e Mathias ${ }^{42}$, que observaram um maior percentual da forma virchowiana. Não foi verificada associação significativa entre a classificação da hanseníase e o tempo em que o paciente era portador da doença e maior necessidade de tratamento odontológico.

As manifestações da hanseníase na mucosa oral estão geralmente relacionadas às formas mais avançadas da doença. São mais comuns na forma virchowiana, indicando uma manifestação tardia e que têm uma grande importância epidemiológica como fontes de infecção ${ }^{23}$. Neste estudo, entretanto, não foi verificada correlação entre a autopercepção da necessidade de tratamento odontológico e a manifestação clínica da hanseníase.

Foi observada associação entre a autopercepção da necessidade de tratamento e a classificação da saúde bucal e a aparência dos dentes e gengivas. A pior percepção da saúde bucal e da aparência dos dentes e gengivas fez com que os entrevistados sentissem maior necessidade de realizar tratamentos odontológicos, corroborando o estudo de Helft et al. ${ }^{27}$, Martins et al. ${ }^{34} \mathrm{e}$ Ekanayake e Perera ${ }^{43}$, que também verificaram tal associação.

Diversas pesquisas observaram um predomínio da autopercepção positiva em relação à saúde bucal ${ }^{26-29}$, fato também verificado entre os portadores de hanseníase, o que não necessariamente equivale a uma boa saúde bucal no que se refere aos aspectos clínicos desses pacientes.

Realizando-se um comparativo com os dados do referido estudo e os obtidos no levantamento epidemiológico SB Brasil $(2004)^{36}$, levantamento este que relata um perfil da população brasileira, tem-se que 40,95\% dos entrevistados consideraram sua saúde bucal boa, assim como $36 \%$ dos pacientes com hanseníase. Quanto à aparência dos dentes e gengivas, a percepção de ambos os estudos foi similar, sendo boa para $44 \%$ dos pacientes com hanseníase e para $43,56 \%$ dos brasileiros.

A mastigação foi considerada boa para $49 \%$ dos pacientes com hanseníase e para 55,30\% da população brasileira, enquanto a fala foi consi- derada boa para 53\% dos pacientes com hanseníase e $62,53 \%$ dos brasileiros, demonstrando em ambos os casos, que os resultados do SB Brasil foram mais positivos.

Quando avaliado se a saúde bucal afeta o relacionamento interpessoal, verificou-se que a autoestima dos entrevistados encontrava-se comprometida, pois $67 \%$ relataram que afeta de alguma maneira, valor bem diferente do verificado para a população do Brasil, em que 23,60\% tiveram a mesma opinião. É importante ressaltar que na hanseníase o indivíduo encontra-se deveras marginalizado e excluído da sociedade, trazendo prejuízos físicos, mas principalmente, psicológicos ${ }^{1,2,6-10}$. Portanto, o fato de não ter uma saúde bucal adequada torna-se problema de grande importância para a pessoa vivendo com hanseníase.

Referindo-se à presença de dor nos últimos seis meses, dentre os estudados, $58 \%$ não relataram nenhuma, enquanto $66,70 \%$ dos entrevistados no SB Brasil, não sofreram com dores dentárias ou gengivais nos últimos seis meses, o que pode refletir uma pior condição de saúde bucal dos pacientes com hanseníase.

Estudos mostram que pacientes com hanseníase apresentam tendência para uma saúde dental e periodontal deficientes, independentemente do tipo de hanseníase ${ }^{44}$. Segundo Aarestrup et al. ${ }^{19}$, Belmonte et al..$^{25}$ e Nunez-Merti ${ }^{44}$, a saúde bucal dos pacientes com hanseníase é precária, pois estas pessoas, comumente, apresentam elevado índice de CPOD (dentes cariados perdidos e obturados) e sérios problemas periodontais. Deve ser levado em consideração que o perfil social e econômico destes pacientes é precário o que contribui para a elevação do índice de CPOD e doença periodontal.

Apesar da autopercepção da saúde bucal ter sido ótima ou boa para $44 \%$ dos estudados, a maioria (73\%) relatou ter necessidade de executar tratamento odontológico, o que deve ser estimulado, em razão da importância da manutenção de uma saúde bucal livre de focos de infecções, os quais podem comprometer a saúde sistêmica do indivíduo e com isso agravar o desenvolvimento da doença.

O fato de infecções odontológicas causarem reações hansênicas, e com isso agravar os sintomas da doença, deve ser destacado, visto que 58\% dos portadores de hanseníase apresentaram episódios de reações. Com isso, uma atenção maior deve ser dada a infecções orais, visto que a detecção e o tratamento destas podem impedir a exarcebação da doença ${ }^{23}$. 
O acesso ao serviço odontológico foi possível para $98 \%$ dos pacientes com hanseníase, valor um pouco maior do que o da população brasileira investigada no SB Brasil, em que 94,19\% o acessaram $^{36}$. A promoção de saúde, porém, ainda necessita ser aprimorada, visto que apenas $30 \%$ dos entrevistados receberam informações sobre como evitar os problemas bucais.

Para Ekanayake e Perera ${ }^{43}$, a promoção da saúde bucal deve ser integrada às estratégias de promoção de saúde geral. Promover saúde é uma estratégia complexa onde a população deve ser avaliada de maneira holística ${ }^{45}$. A proposta é articular saberes técnicos e populares, com a mobilização de recursos institucionais e comunitários, públicos e privados, para seu enfrentamento e resolução ${ }^{46,47}$.

O prejuízo que a hanseníase trouxe para a saúde bucal mostrou-se correlacionado à dificuldade de realizar a higiene oral. Para Costa et al. $^{23}$, diversos motivos podem dificultar a higiene, como os episódios reacionais em que o paciente fica com sua saúde sistêmica comprometida, tornando mais difícil a execução dos hábitos de higiene corporal, incluindo também a bucal. Além disso, dentre as sequelas da doença, as mãos em garra e as amputações de dedos, algo que só acontece nos casos mais avançados da doença, não submetidos ao tratamento adequado, podem interferir diretamente na saúde oral, pois torna a higiene bucal algo mais complicado de se realizar e deixa o paciente desmotivado a utilizar aparelhos protéticos. Segundo Russo et al. ${ }^{20}$, ocorre um comprometimento psicossocial, gerando indivíduos pouco preocupados com qualidade de vida e com sua saúde bucal.

Este estudo reforça a importância da saúde bucal dos pacientes com hanseníase, que apresentam uma saúde sistêmica comprometida, con- tribuindo para que seja deixada em segundo plano. Alguns fatores devem ser levados em consideração quando se avalia a autopercepção da saúde bucal e da necessidade de tratamento odontológico, como: escolaridade, renda, tipo de hanseníase, presença de reações hansênicas e dificuldade de realizar a higienização dos dentes e gengivas. Além disso, o conhecimento dessas características auxilia na execução de um planejamento em saúde adequado para a assistência desses pacientes.

A autopercepção da pessoa com hanseníase quanto à sua saúde bucal e necessidade de tratamento deve, juntamente com uma avaliação clínica, servir como guia para a execução de políticas públicas que visem a favorecer um tratamento odontológico mais efetivo para esses pacientes.

Dessa forma, a execução de programas que promovam a saúde bucal em hanseníase deve ser estimulada, buscando-se desta maneira proporcionar uma atenção integral á saúde destas pessoas.

\section{Conclusões}

Neste estudo, a autopercepção da pessoa vivendo com hanseníase quanto à sua necessidade de tratamento odontológico foi influenciada diretamente pela escolaridade.

Pessoas que autopercebem a saúde bucal como péssima, ruim e regular acreditam ter maior necessidade de realizar tratamento odontológico.

Outro fator importante na autopercepção da necessidade de tratamento foi a aparência dos dentes e gengivas, pois uma pior aparência reflete maior necessidade de realizar tratamento.

A maioria destes pacientes acredita que a referida doença não trouxe prejuízo para sua saúde bucal.

\section{Colaboradores}

JRS Almeida trabalhou na concepção e projeto, análise e interpretação dos dados e redação do artigo. CH Alencar trabalhou na análise e interpretação dos dados. MEL Almeida trabalhou na concepção e projeto, Revisão crítica relevante do conteúdo intelectual, Aprovação final da versão a ser publicada. JC Barbosa e AA Dias trabalharam na revisão crítica relevante do conteúdo intelectual. 


\section{Referências}

1. Santos AS, Castro DS, Falqueto A. Fatores de risco para transmissão da Hanseníase. Rev Bras Enferm 2008; 61(Esp.):738-743.

2. Borenstein MS, Padilha MI, Costa E, Gregório VRP, Koerich AME, Ribas DL. Hanseníase: estigma e preconceito vivenciadas por pacientes institucionalizados em Santa Catarina (1940-1960). Rev Bras Enferm 2008; 61(Esp.):708-712.

3. Brasil. Ministério da Saúde (MS). Secretaria de Vigilância em Saúde. Departamento de Vigilância Epidemiológica. Manual de Prevenção de Incapacidades. 3a Edição, Revisada e ampliada. Brasília: MS; 2008.

4. Santos AK, Monteiro S, Rozemberg B. Significados e usos de materiais educativos para hanseníase segundo profissionais de saúde pública do Município do Rio de Janeiro, Brasil. Cad Saude Publica 2009; 25(4):857-867.

5. Oliveira MHP, Romanelli G. Os efeitos da hanseníase em homens e mulheres: um estudo de gênero. Cad Saude Publica 1998; 14(1):51-60.

6. Nunes JM, Oliveira EM, Vieira NFC. Ter Hanseníase: percepções de pessoas em tratamento. Rev Rene 2008; 9(4):91-98.

7. Eidt LM. Breve história da hanseníase: sua expansão do mundo para as Américas, o Brasil e o Rio Grande do Sul e sua trajetória na saúde pública brasileira. Rev Saúde Sociedade 2004; 13(2):76-88.

8. Pontes ARB, Almeida MGC, Xavier MB, Quaresma AS, Yassei EA. Detecção do DNA de Mycobacterium leprae em secreção nasal. Rev Bras Enferm 2008; 61(Esp.):734-737.

9. Vieira CSCA, Soares MT, Ribeiro CTSX, Silva LFG. Avaliações e controle de contatos faltosos de doentes com hanseníase. Rev Bras Enferm 2008; 61(Esp.): 682-688.

10. Silva Júnior FGJG, Ferreira RD, Araújo OD, Campêlo SMA, Nery IS. Assistência de enfermagem ao portador de Hanseníase: abordagem transcultural. Rev Bras Enferm 2008; 61(Esp.):713-717.

11. Façanha MC, Pinheiro AC, Lima JRC, Ferreira MLLT, Teixeira GFD, Rouquayrol MZ. Hanseníase: subnotificação de casos em Fortaleza - Ceará, Brasil. An Bras Dermatol 2006; 81(4):329-333.

12. Nations MK, Lira GV, Catrib AMF. Estigma, metáforas deformadoras e experiência moral de pacientes com hanseníase multibacilar em Sobral, Ceará, Brasil. Cad Saude Publica 2009; 25(6):1215-1224.

13. Costa LCV, Andrade KLC, Carmo MAV, Ferreira MAA, Garrocho AA. Manifestações Bucofaciais da Hanseníase. Rev CROMG 2002; 8(3):191-197.

14. Ranjan KB, Belliappa PR, Ebenezer G, Job CK. Single lesion borderline lepromatous leprosy. Int J Lepro Other Mycobact Dis 2004; 72(1):45-47.

15. Moreno CMC, Enders BC, Simpson CA. Avaliação das capacitações de Hanseníase: opinião de médicos e enfermeiros das equipes de saúde da família. Rev Bras Enferm 2008; 61(Esp):671-675.

16. Diallo B, Bourgeois D, Coudert JL. Evolution of the orofacial and dental status of a population of leprosy patients treated with multidrug therapy in Senegal. Acta Leprol 1992; 8(1):11-15.
17. Belo Horizonte. Secretaria do Estado de Saúde de Minas Gerais (SESMG). Como reconhecer e tratar reações hansênicas. Belo Horizonte: SESMG; 2007.

18. Cortela DCB, Ignotti C. Conhecimento e experiências do cirurgião dentista sobre hanseníase em Cáceres, MT, Brasil. Rev Odonto Ciênc 2008; 23(3):243250.

19. Aarestrup FM, Aquino MA, Castro JM, Nascimento DN. Doença periodontal em hansenianos. Rev Perio 1995; 4(1):191-193.

20. Russo MP, Corrêa CT, Martins MAT, Martins MD. Aspectos da doença de Hansen relevantes para o cirurgião-dentista: revisão da literatura. Rev Odonto Ciência 2005; 20(48):126-131.

21. Abreu MAMM, Michalany NS, Wechx LLM, Pimentel DRN, Hirata CHW, Alchorne MMA. The oral mucosa in leprosy: a clinical and histopathological study. Rev Bras Otor 2006; 72(3):312-316.

22. Motta ACF, Komesu MC, Silva CHL, Arruda D, Simão JCL, Zenha EMR, Furini RB, Foss NT. Leprosy-specific oral lesions: A report of three cases. Med Oral Patol Oral Cir Bucal 2008;13(8):479-482.

23. Costa APF, Nery JAC, Oliveira MLW, Cuzzi T, Silva MR. Oral lesions in leprosy. Indian Journal of Dermatology, Venereology and Leprology 2003; 69(6):381385.

24. Martins MD, Russo MP, Lemos JBD, Fernandes KPS, Bussadori SK, Corrêa CT, Martins MAT. Orofacial lesions in treated southeast Brazilian leprosy patients: a cross-sectional study. Oral Diseases 2006; 13(3):270-273.

25. Belmonte PCR, Tonello AS, Virmond MCL, Belmonte GC, Monti JFC. Características da doença periodontal em hanseníase. BEPA. Boletim Epidemiológico Paulista 2007; 4(44):4-9 .

26. Martins AMEBL, Barreto SM, Pordeus IA. Autoavaliação de saúde bucal em idosos: análise com base em modelo multidimensional. Cad Saude Publica 2009; 25(20):421-435.

27. Helft MW, Gilbert GH, Shelton BJ, Ducan RP. Relationship of dental status, sociodemographic status, and oral symptoms to perceived need for dental care. Community Dent Oral Epidemiol 2003. 31(5):351-360

28. Benyamini $\mathrm{Y}$, Leventhal $\mathrm{H}$, Leventhal EA. Self rated oral health as an independent predictor of self rated general health, self esteem and life satisfaction. Soc Sci Med 2004; 59(5):1109-1116.

29. Silva SRC, Fernandes RAC. Autopercepção das condições de saúde bucal por idosos. Rev Saude Publica 2001; 35(4):349-355

30. Mathias RE, Atchison KA, Lubben JE, De-jong F, Schweitzer SO. Factors affecting self-ratings of oral health. J Public Health Dent 1995; 55(4):197-204.

31. Silva DD, Sousa MLR, Wada RS. Autopercepção e condições de saúde bucal em uma população de idosos. Cad Saude Publica 2005; 21(4):1251-1259.

32. Reis SCGB, Marcelo VC. Saúde bucal na velhice: percepção dos idosos, Goiânia, 2005. Cien Saude Colet 2006; 11(1):191-199. 
33. Locker D, Clarke M, Payne B. Self perceived oral health status, psychological well-being, and life satisfaction in an older population. J Dent Res 2000; 79(4):970-975.

34. Martins AMEBL, Barreto SM, Pordeus IA. Fatores relacionados à auto-percepção da necessidade de tratamento odontológico entre idosos. Rev Saude Publica 2008; 42(3):487-496.

35. Brasil. Ministério da Saúde. Conselho Nacional de Saúde. Resolução nº 196 de 10 de outubro de 1996. Diretrizes e Normas Regulamentadoras de Pesquisas Envolvendo Seres Humanos. Diário Oficial da União 1996; out 16.

36. Brasil. Ministério da Saúde (MS). Coordenação Nacional de Saúde Bucal. Resultados principais do projeto SB Brasil 2003. Condições de saúde bucal da população brasileira 2002-2003. Brasília: MS; 2004.

37. Duarte MTC, Ayres JA, Simonetti JP. Socioeconomic and demographic profile of leprosy carriers attended in nursing consulatations. Rev Latino-am Enfermagem 2007; 15(Esp.):774-779.

38. Cunha AZS. Hanseníase: aspectos da evolução do diagnóstico, tratamento e controle. Cien Saude Colet 2002; 7(2):235-242.

39. Santana SC, Ueda ES, Schrcuder PAM, Gonide M. Papel das ações educativas e o controle da hanseníase no município de Ariquemes, Rondônia. Cad Saude Coletiva 2008; 16(2):181-192.

40. Amaral EP, Lana FCF. Análise espacial da Hanseníase na microrregião de Almenara, MG, Brasil. Rev Brasil Enferm 2008; 61(Esp.);801-807.

41. Kerr-Pontes LRS, Montenegro ACD, Barreto ML, Werneck GL, Felmeier H. Inequality and leprosy in Northeast Brazil: an ecological study. Int J Epidemiol 2004; 33(2):262-269.

42. Sobrinho RAS, Mathias TAF. Perspectivas de eliminação da hanseníase como problema de saúde pública no Estado do Paraná, Brasil. Cad Saude Publica 2008; 24(2):303-314.

43. Ekanayake L, Perera I. Perceived need for dental care among dentate older individuals in Sri Lanka. Spec Care Dentist 2005; 25(4):199-205.

44. Nunez-merti JM, Bagan JV, Scully C, Penarrocha M. Leprosy: dental and periodontal status of the anterior maxilla in 76 patients. Oral Diseases 2003; 10(1):19-21

45. Maltz M, Jobim JJ, Alves LS. Health promotion and dental caries. Braz Oral Res 2010; 24(Spec Iss 1):18-25.

46. Moretti AC, Teixeira FF, Suss FMB, Lawder JAC, Lima LSM, Bueno RE, Moyses SJ, Moyses ST. Intersetorialidade nas ações de promoção de saúde realizadas pelas equipes de saúde bucal de Curitiba (PR). Cien Saude Colet 2010; 15(Supl. 1):1827-1834.

47. Buss PM. Promoção da saúde e qualidade de vida. Cien Saude Colet 2000; 5(1):163-177. 